\title{
Exploración de los Derechos Sexuales y Reproductivos: conceptos y Elementos Claves ${ }^{1}$
}

\author{
Miryam Cristina Fernández Cediel \\ Universidad Surcolombiana \\ cristina.fernandez@usco.edu.co \\ Roberto Cortes \\ Universidad Surcolombiana
}

Sebastián Cachaya
Camila González
Carolina Merchán
Lina Motta
Mila Dahlia
Yesenia López
Yina Muñoz
Hernán Cáceres
Leandro Gaviria
Clara Rivera

Universidad Surcolombiana

\section{Resumen}

Ante las múltiples polémicas que Latinoamérica vive en asuntos relacionados a los derechos sexuales y reproductivos ya sea en lo legislativo por la aprobación del aborto o el aumento de penas por crímenes contra la mujer, o en lo cultural donde se promueve el machismo y se culpa a las mujeres de sus propias lesiones o muertes, es necesario promover la comprensión y transformación de estos fenómenos. Este documento tiene el propósito de mostrar el avance en la construcción del estado de arte de estos derechos en la comunidad científica a partir de la búsqueda de la producción escrita en las bases de datos de Latinoamérica. Se encontró que el reconocimiento de estos derechos surge a partir de distintas conferencias que promueven los derechos de la mujer, que el avance en el ejercicio de estos derechos ha sido obstaculizado por la cultura patriarcal y la religión católica, que el entorno educativo juega un rol fundamental para que adolescente y jóvenes asuman sus derechos. La comunidad científica debe apoyar estos procesos de reconocimiento y ejercicio de los derechos sexuales y reproductivos.

Palabras Clave: Derechos sexuales y reproductivos, mujer, adolescentes, educación.

1 Este artículo fue escrito en el marco de formación de estudiantes en investigación y proyección social del grupo CRECER de la Universidad Surcolombiana. La elección de esta temática contribuye a las bases conceptuales del proyecto "Derechos sexuales y reproductivos en familias en situación de desplazamiento en la ciudad de Neiva" que se desarrolla con la participación de todos los autores de este documento 


\title{
Exploration of Sexual and Reproductive Rights: Concepts and Key Elements
}

\begin{abstract}
Given the many controversies that Latin America lives in issues related to sexual and reproductive rights, whether in the legislature for the approval of abortion or increased penalties for crimes against women, or in the cultural where it is promoted machism and women are blamed for their own injuries or deaths, it is necessary to promote the understanding and transformation of these phenomena. This document is intended to show progress in the construction of the state of art of these rights in the scientific community from the search for written production in the Latin American databases. It was found that the recognition of these rights arises from different conferences that promote the rights of women, that progress in the exercise of these rights has been hampered by the patriarchal culture and the Catholic religion, that the educational environment plays a role fundamental for adolescents and young people to assume their rights. The scientific community should support these processes of recognition and exercise of sexual and reproductive rights.
\end{abstract}

Keywords: Sexual and reproductive rights, women, adolescents, education.

\section{Introducción}

Los derechos sexuales y reproductivos (DSR) están vinculados a los derechos universales, son esenciales a todos los seres humanos que tienen en cuenta la parte biológica, psicológica, cultural, histórica y ética del ser humano (Bedoya Abella, 2014). Están basados en la libertad, dignidad e igualdad inherentes a todos los seres humanos y tienen directa relación con los derechos a: la vida, la supervivencia, la seguridad y la sexualidad; la autodeterminación reproductiva y la libre opción de la maternidad, la salud y los beneficios del progreso científico; la no discriminación y el debido respeto por las diferencias; la información, la educación y la toma de decisiones. Inciden en la defensa de la sexualidad y como todos los derechos, éstos cobijan al ser humano desde el nacimiento y hacen referencia a aquellos aspectos como el embarazo, la intimidad, el género, el sexo, la orientación sexual, la información y educación (Villanueva Flores, 2006).

El ejercicio de los DSR implica el bienestar físico, emocional y social, en especial, de las mujeres en todos los aspectos relacionados con la sexualidad y la reproducción, esto es: disfrutar de una vida sexual satisfactoria $y$ sin riesgos, ejercer el derecho a procrear o no, libertad para decidir el número y espaciamiento de los hijos, obtener información que posibilite la toma de decisiones libres e informadas y sin sufrir discriminación, coerción, ni violencia, acceder y elegir métodos de regulación de la fecundidad seguros, eficaces y aceptables, eliminar la violencia doméstica y sexual, recibir servicios adecuados de atención a la salud y el acceso a servicios y programas de calidad para la promoción, detección, prevención y atención de todos los eventos relacionados con la sexualidad y la reproducción, independientemente del sexo, edad, etnia, clase, orientación sexual o estado civil (Bonaccorsi \& Reybet, 2008; Ciriza, 2002).

Los derechos sexuales y reproductivos forman parte de los derechos humanos, y su base se sustenta en los valores: dignidad, igualdad y libertad (Távara Orozco, 2016). Esto implica que, por un lado, tengan una connotación universal, en donde todas las personas desde el momento del nacimiento estén cobijadas por ellos y por ende sean inalienables. Por otro lado, aunque exista el reconocimiento de los derechos humanos y los DSR en algunos sectores de la sociedad, existen deficiencias y enormes brechas de desinformación en los sectores más vulnerables de la población. Esta desinformación, sumada a diferentes factores como la fuerte influencia de la cultura patriarcal, la influencia religiosa sobre la sexualidad, los imaginarios sociales conservadores, los bajos recursos, la violencia, conlleva a que estos derechos sean violados e invisibilizados y como 
resultado haya muy poco empoderamiento de los mismos (Mayorga Madrigal, 2016; Valdés Díaz, 2012; Zicavo, Astorino, \& Saporosi, 2015).

Estos derechos son relevantes porque representan un pilar para el ejercicio de la ciudadanía, pues a partir de estos derechos, las personas estarán en capacidad de tomar decisiones autónomas en todas las áreas de su ser, incluidas la sexualidad y la reproducción.

El Instituto Colombiano de Bienestar Familiar [ICBF] (2008) en su módulo conceptual sobre los derechos sexuales y reproductivos manifiesta que "la sexualidad es una construcción social simbólica, hecha a partir de una realidad propia de las personas: seres sexuados en una sociedad determinada" (p. 16). Los DSR favorecen el entendimiento y apropiación de la sexualidad en los individuos debido a que permiten la defensa de las elecciones personales sobre el cuerpo y la sexualidad para el pleno goce de esta.

Este artículo expone elementos claves rescatados en el proceso de construcción del estado de arte sobre los DSR.

\section{Método}

Este escrito reporta el primer avance en el proceso de construcción del estado de arte sobre derechos sexuales y reproductivos, para ello dentro de la base de sistematización de la información encontrada se registraron 215 documentos que fueron localizados en las bases de datos de Science Direct, Dialnet, Redalyc, Clacso y Scielo, a través de las palabras de búsqueda "derechos sexuales y reproductivos". Para la elaboración de este artículo se recuperaron los registros que se reportan en la tabla 1.

Tabla 1. Relación de Documentos Encontrados en las Bases de Datos Seleccionadas.

\begin{tabular}{ccc}
\hline Bases de datos & Número de documentos & Porcentaje \\
\hline Science Direct & 3 & 1,4 \\
Dialnet & 82 & 38,1 \\
Redalyc & 73 & 34 \\
Clacso & 36 & 16,7 \\
Scielo & 21 & 9,8 \\
\hline
\end{tabular}

De todos los 215 documentos 164 fueron artículos publicados en revistas científicas (76\%), 21 fueron capítulos de libros $(9,7 \%), 4$ eran libros $(1,9 \%)$ y 26 fueron otro tipo de títulos de divulgación.

Entre los criterios establecidos para la revisión documental se encontraban artículos que estuvieran publicados entre el 2006 y el 2016, que fueran escritos en español, que dieran cuenta de la producción latinoamericana y por último que hicieran un especial énfasis sobre los DSR.

Bajo estos parámetros establecidos se seleccionaron 94 artículos para la revisión y análisis de contenidos y 122 artículos se rechazaron por no cumplir los criterios de inclusión. Sin embargo, dentro del proceso de refinación de los hallazgos se encontró que varios artículos fueron publicados en distintas bases de datos, por ello se redujeron a 74 documentos para la revisión: 9 de Argentina, 2 de Bolivia, 1 de Brasil, 5 de Chile, 16 de Colombia, 8 de Costa Rica, 1 de Cuba, 1 de España, 18 de México, 9 de Perú, 2 de Uruguay y 2 de Venezuela.

\section{Resultados}

A continuación, se presentan los principales hallazgos que se discuten en los artículos revisados.

\section{Elementos Históricos de los DSR}

La comunidad internacional comenzó a esbozar los conceptos de derechos reproductivos como fundamento de las intervenciones en el comportamiento reproductivo de la población. Es así como en la Conferencia Mundial de los Derechos Humanos celebrada en Teherán (1968), se sientan las bases para el progresivo reconocimiento y desarrollo de los DSR al afirmar por primera vez que son las personas las que deben tomar las decisiones acerca del número y espaciamiento de los hijos que desean tener. Posteriormente, en la Conferencia Mundial de Población de Bucarest (1974) se reafirmó el carácter de derecho fundamental de las decisiones reproductivas y se reconoce que es un derecho de los individuos (no de las 
parejas, ni exclusivo de los padres) decidir sobre la regulación de la fecundidad. Igualmente se enfatiza en la responsabilidad del Estado de proporcionar información, educación, y los medios que permitan a los individuos el ejercicio de tal derecho.

A partir de 1979 con la Convención para la Eliminación de Todas las Formas de Discriminación Contra la Mujer (CEDAW) se cuenta con el primer instrumento internacional con perspectiva de género que propone un cambio en la concepción de los derechos de las mujeres. La declaración en mención es un ejemplo de la evolución de los derechos orientada a la configuración de las mujeres como sujetos titulares de derechos.

Desde entonces, la comunidad internacional ha llegado a nuevos consensos en relación con el reconocimiento de estos derechos que deben ser acompañados de la voluntad política necesaria para que puedan ser efectivamente ejercidos por todas las personas, sin distinciones ni discriminaciones en virtud de sexo, edad, condición socioeconómica, religión, origen racial, estado civil, idioma o cualquier otra condición. En este progresivo proceso de reconocimiento de los Derechos Sexuales y Reproductivos han sido fundamentales:

a) La II Conferencia Mundial sobre la Mujer, en Copenhague, en la que se aprueba el programa de acción para la segunda mitad del Decenio de la Mujer;

b) La III Conferencia Mundial en Nairobi (1985) sobre la Mujer en la cual se evalúa el Decenio y se aprueba por consenso el documento "Las Estrategias de Nairobi", orientado hacia el futuro y para el adelanto de las mujeres por los próximos 15 años (hasta el 2000);

c) La Conferencia Mundial de los Derechos Humanos en Viena (1993) en la que la comunidad internacional declara y reconoce por primera vez que "los derechos humanos de la mujer y de la niña son parte inalienable, integrante e indivisible de los derechos humanos universales", también se refiere al derecho de todas las personas a tener el control sobre su sexualidad y a vivirla plenamente decidiendo libre y responsablemente, y a la capacidad de disfrutar de una vida sexual satisfactoria y sin riesgos, la de procrear y la libertad de decidir hacerlo o no, conllevan a un entramado de derechos como el de la información, la libertad, la seguridad, la igualdad, entre otros se ha hecho difícil su ejercicio y aun no se garantiza el goce de estos (Cruz Pérez, 2015; Staff Wilson, 1998; Valdés Díaz, 2012).

d) Los DSR fueron reconocidos en la Conferencia Internacional de Población y Desarrollo en el Cairo en 1994 y desde ese entonces se ha emprendido un movimiento en los gobiernos y la comunidad en general para trasformar y trascender en las formas de ver y comprender el mundo de los derechos en especial los sexuales y reproductivos, y así poder fomentar la conciencia y autonomía en hombres y mujeres sobre la responsabilidad que acuña su cuerpo y sus formas de relacionarse en la sociedad (Carreño Ruiz, Artunduaga Alpire, Suarez Barrientos, \& Cornejo Bascopé, 2007).

e) La IV Conferencia Mundial de la Mujer (Beijing 1995) durante la cual se aprueba la Declaración de Beijing que compromete a los gobiernos a impulsar, antes del término del siglo XX, las estrategias acordadas en Nairobi en 1985 y a movilizar recursos para la realización de la plataforma. La Plataforma de Acción de Beijing es el documento más completo producido por una conferencia de Naciones Unidas en relación con los derechos de las mujeres, ya que incorpora lo logrado en conferencias y tratados anteriores.

No obstante, el término, 'Derechos sexuales' no es usado en el Programa de Acción de El Cairo ni en la Plataforma de Acción de Beijing. Sin embargo, en esta última se señala que los derechos humanos de la mujer incluyen su derecho a tener control sobre las cuestiones relativas a su sexualidad, incluida su salud sexual y reproductiva, y decidir libremente respecto de esas cuestiones, sin verse sujeta a la coerción, la discriminación y la violencia.

En América Latina, las Conferencias Regionales realizadas periódicamente desde hace tres décadas, han sido fundamentales en la generación de consensos en torno a los derechos humanos de las mujeres, incluidos los derechos 
sexuales y reproductivos, y para la adopción de políticas para su protección. Así, el Consenso de Santiago (1997) invitó a formular programas que protegieran los DSR de las mujeres a través de información, educación y servicios que ayuden sobre la salud sexual y reproductiva. El Consenso de Lima (2000) hace un llamado para atender las violaciones de los DSR además de mantener la invitación realizada en Santiago. El Consenso de México (2004) enfatiza en la dimensión étnicoracial y la diversidad existente en la región, así como a la dimensión generacional.

En el caso de los Derechos Reproductivos, Corrêa \& Petchesky (1996) afirman que el término se empleó por primera vez en Estados Unidos en 1979, con la creación de la Red Nacional de Derechos Reproductivos. Empero, es a partir de la $\mathrm{V}$ Conferencia Internacional sobre Población y Desarrollo (CIPD) que el uso del término "derechos reproductivos" empezó a generalizarse para reivindicar los derechos que tienen las mujeres en el ámbito reproductivo.

La Conferencia Mundial realizada en Beijing expresa que los derechos reproductivos se relacionan con el derecho a elegir el número de hijos, el espaciamiento de los nacimientos y el intervalo entre éstos y a disponer de la información y de los medios para ello y el derecho a alcanzar el nivel más elevado de salud sexual y reproductiva. Además, sugiere que los estados parte adopten medidas que permita eliminar la discriminación contra la mujer.

En cuanto a textos constitucionales, debe señalarse que los artículos 36 y 43 de la Carta ecuatoriana de 1998, menciona explícitamente los derechos reproductivos y la salud sexual y reproductiva; también lo hace la Constitución de la Ciudad de Buenos Aires de 1996; el derecho a decidir sobre el número de hijos y su espaciamiento está reconocido en las constituciones de Brasil, México, Colombia, Ecuador, Perú y Paraguay (Montaño \& Aranda, 2006; Straw \& Mattioli, 2013). Se suele incluir en la lista de derechos reproductivos la atención de la mujer durante el embarazo y el parto y la reproducción asistida. En cuanto a la inclusión de la interrupción del embarazo en la lista de derechos reproductivos, este es un tema controversial. De un lado, hay recomendaciones del Comité de Naciones Unidas para revisar la legislación interna en materia de represión penal del aborto, pues se considera que vulnera el Pacto Internacional de Derechos Civiles y Políticos. Por otra parte, en Estados Unidos y España, la despenalización del aborto ha tenido como eje principal la defensa de los derechos a la intimidad, al libre desarrollo de la personalidad y a la dignidad personal de la mujer.

Aunque los textos constitucionales mencionados y la definición contenida en el Programa de Acción de la CIPD contemplan que los derechos reproductivos protegen tanto a hombres como a mujeres, es usual considerarlos como especiales para las mujeres en razón a las responsabilidades asignadas culturalmente en esta materia (Bareiro, 2003).

La protección constitucional de los DSR es de vital importancia particular en tanto su ejercicio está ligado a la esfera privada en donde ocurren con mayor frecuencia las vulneraciones a los derechos de las mujeres. Durante mucho tiempo tales afectaciones no fueron objeto de preocupación de los estados, lo cual se expresa en la ausencia de mecanismos legales de protección de las mujeres frente a la violencia doméstica y de garantía de su autonomía en relación a su propia fecundidad y su corporalidad (Villanueva Flores, 2006).

Como producto de tales desarrollos, en la actualidad se reconoce en la legislación colombiana, por ejemplo, la existencia de derechos sexuales y reproductivos que representan el pilar fundamental para el ejercicio de la ciudadanía, entendida más allá de la posibilidad de tomar decisiones en el ámbito público (elegir y ser elegido); en tanto implica la posibilidad de tomar decisiones autónomas sobre su cuerpo y su vida en los campos de la sexualidad y la reproducción.

A pesar de tales reconocimientos legislativos son múltiples las situaciones que expresan la existencia y pervivencia de una cultura patriarcal que no permite la realización y ejercicio de tales derechos, como se ha observado en los debates 
recientes en torno al tema del aborto, la unión de parejas homosexuales y la adopción, así como la naturalización de la violencia hacia las mujeres y el notorio incremento del abuso sexual y los embarazos precoces e indeseados (Petracci \& Pecheny, 2009; Romero Ricalde, 2007). A esto se suma que los derechos sexuales y reproductivos aún no han sido reconocidos a cabalidad como derechos fundamentales y no han sido incorporado aun en todas las constituciones (Valdés Díaz, 2012), aun cuando la Conferencia de Naciones Unidas y las diversas convenciones hayan generado los recursos jurídicos necesarios para garantizar la implementación de estos derechos, éstos no se concretan en la práctica, ya que con frecuencia las instituciones se ven permeadas de los prejuicios de sus propios funcionarios (Cruz Pérez, 2015).

\section{Los DSR y la Cultura}

Los DSR afectan $y$ se transforman significativamente en la política, la cultura, la economía, las tecnologías, entre otros contextos. Las relaciones que se desarrollan con el entorno (social, ambiental, cultural) determinan gran parte del sentido que se le otorga a las vivencias y prácticas cotidianas. Las prácticas sexuales y el contenido subjetivo sobre su cuidado, viene determinado por los elementos históricos de índole personal y grupal, generacional, comunitarios, compuesto por el proceso de socialización y culturización del individuo (Reartes, 2011). Es preciso reconocer que el tema de los derechos sexuales tiene una serie de implicaciones que desafían en gran parte nuestros horizontes culturales (Miller, 2002).

El ejercicio de estos derechos no ha sido tarea fácil, especialmente en una cultura que es permisiva ante la violencia intrafamiliar, la marginación y la estigmatización. Las posturas machistas y privilegios sociales que tiene el hombre afectan directamente en el ejercicio de los DSR de las mujeres, más aún cuando estas lógicas fueron y siguen siendo reforzadas a nivel cultural, histórico y religioso.

Para la época en que los organismos internacionales postulaban políticas a favor de la salud sexual y reproductiva, las disputas no se hicieron esperar, las políticas regionales y patriarcales de la iglesia iban en contra de la planificación y el dejamiento del poder conyugal ejercido por el hombre (Caneva, 2015). Sin embargo, ya desde la década de los sesenta el modelo conyugal y de familia empezó a presentar rupturas y continuidades en toda Latinoamérica. El paso de conservador a un modelo más libertario que pretende que la relación varón - mujer sea menos subordinada a la patriarcalidad y los roles desempeñados por ambos cambien, permite que la mujer desempeñe otros roles diferentes a la domesticidad. Cabe resaltar que para estas épocas, la percepción hacia el matrimonio cambia y el divorcio y la unión libre pasa a ser más aceptado en la cultura de la región (Cosse, 2008).

El reconocimiento pleno de los DSR supone también distanciamiento con los preceptos de la comunidad católica de extrema, ya que estos derechos atentan contra la patriarcalidad con la que esta instaurada su doctrina; pues se basa en la domesticidad de la mujer y la dominancia del hombre como sinónimo de la iglesia en control de los devotos, la jerarquización es fundamental en la estructura religiosa, por ende no es posible perder la dominancia que ejercen (Carbonelli, 2015; Jones \& Carbonelli, 2012; Valencia Jiménez \& Solera Martínez, 2009).

La iglesia católica es una de las primeras en impugnar las políticas que se designan para una formación acerca de la sexualidad; restringe a los niños y niñas de recursos fundamentales para el desarrollo de sus vidas, como lo es un conocimiento adecuado acerca de los DSR los cuales los pondrían en una posición menos vulnerable en actos de violencia sexual por parte de mayores, casos de vulneración de estos derechos que incluso representantes la iglesia han protagonizado como verdugos (Petracci \& Pecheny, 2009). Esta impugnación es resguardada con respuesta como que en Gálatas 6:5 la biblia dice que cada uno llevara su propia carga de responsabilidades, por lo cual se cree que cada persona se debe resolver incluso preguntas personales e íntimas evaluando como lleva su camino por la santidad de la vida (Villanueva Flores, 2006). 
Empoderarse de los DSR no es tarea fácil, el vivir en una sociedad que excluye por ser indígena, ser negro, o ser diferente. Un ejemplo representativo es el que involucra a las comunidades indígenas, al no saber intervenir en ellas, se permiten y aceptan estilos de vida que ponen en riesgo la salud de las personas que las conforman, prácticas como el no usar condón porque el sexo es con fines reproductivos y una forma de comprobar la fidelidad de la pareja, aceptar que hombres roben mujeres muy jóvenes y vírgenes para conformar una familia, da paso a que en su inexperiencia se maltrate a las mujeres, abusen sexualmente de ellas y terminen por ser prisionera en su propio hogar (Hernández-Rosete, García, Bernal, Castañeda, \& Lemp, 2008).

También, estarcontagiadodeVIH, creabarreras entre unos y otros. En el caso de las personas contagiadas con VIH, ellas experimentan las mismas necesidades sexuales y reproductivas que las personas que no lo están; no obstante, la búsqueda de la satisfacción de éstas es más complejo para ellas por la estigmatización y la creencia de que cualquier persona que los toque también será contagiado (Campero, Kendall, Caballero, Mena, \& Herrera, 2010).

Por su parte, las personas con discapacidad que frecuentemente se las califica de improductivas, disminuidos e inferiores también se les niega la promoción y protección de la dignidad humana. Ellas han sido a quienes más se les han violado sus DSR por la creencia de que estas son dependientes y asexuadas. Su libertad y autonomía quedan restringidas a las decisiones de sus cuidadores y que debido al desconocimiento de sus derechos con frecuencia estas personas se ven obligadas a procedimientos de esterilización o aborto sin su consentimiento (Cruz Pérez, 2015).

El ejercicio adecuado de los DSR tiene fuertes implicaciones sobre la salud. Távara (2016) afirma que "muchos de los problemas de salud relacionados al sexo o a la sexualidad dependen de la naturaleza de las relaciones de poder entre hombres y mujeres" (p. 34). Partiendo de esta premisa, aunque actualmente algunos movimientos sociales como los movimientos feministas luchen por la reivindicación de los derechos de las mujeres (incluyendo los sexuales y reproductivos), la balanza parece seguir estando del lado del hombre (D. González, 2009).

El resultado de la violación de los DSR en las mujeres puede variar, pero no por ello ser menos perjudicial para la salud, estas consecuencias pueden ser: abortos inseguros, imposición del número de hijos, no elegir ni decidir libremente sobre su sexualidad, discriminación, ITS, violencia física, psicológica y sexual y en los peores de los casos la muerte. La violencia basada en el género, como se mencionó anteriormente, esta reforzada por diferentes aspectos culturales, de orden religioso, y modelos e imaginarios tradicionales y conservadores sobre lo que debe hacer un "macho" y una concepción de que la mujer es un objeto sin derechos, no es libre de decidir sobre su cuerpo, sobre su sexualidad y sobre sus propios derechos (A. C. González, 2016; A. González \& Victoria, 2012; Távara Orozco, 2016).

Además, las creencias y prácticas sexuales de los adolescentes se fundamentan en la educación que han recibido de sus padres y docentes, y estos provienen de un método de aprendizaje en el cual se prepara a la mujer para una vida de sumisión, con una vida sexual ligada a las emociones y sentimientos y a los hombres con criterios de hombría, viendo tanto al sexo como a la sexualidad como fuentes de placer y descargas físicas.

A pesar de esta fuerte tendencia, el feminismo contemporáneo denuncia la obligatoriedad de la maternidad y la heteronormatividad, y propone repensar los mandatos sociales y separar la sexualidad de la reproducción y dejar de ver la maternidad como un objetivo de vida. Esta propuesta sigue teniendo sus detractores que cuentan con aliados entre los decisores políticos, que en gran mayoría se oponen al avance de la mujer, por considerarlo amenazante con el tipo de vida normal en tópicos familiares y sexuales (Bonaccorsi \& Reybet, 2008; Petracci \& Pecheny, 2009).

Cabe aclarar que en lo que se encuentra en la literatura por lo general se asume que los DSR 
solo pertenecen a las mujeres, reconociendo a los hombres como garantes de estos en contadas excepciones y condiciones. Los avances en la elaboración de estrategias de inclusión son útiles y convenientes a la sociedad para no pasar por encima de los derechos de los demás.

Deben existir políticas de salud pública, en las cuales sehagan valer los DSR, desdela perspectiva de calidad de los usuarios en los servicios como planificación familiar, control prenatal, abortos, psicoeducación, entre otros. Todo esto con el fin de propiciar una identificación principalmente de las necesidades en la población, y asegurando así la integralidad de la atención y el constante restablecimiento de los DSR cuando haya lugar (Pacheco-Sánchez et al., 2007).

\section{Las Mujeres y los DSR}

Es importante reconocer a las mujeres como sujetos activos y participativos. A través de la historia se da cuenta que ellas buscan reconocimiento y cumplimiento a sus derechos, debido a que la sociedad mantiene una situación de desventaja con las mujeres en relación a los hombres. En este trayecto de reconocimiento de sus derechos, las mujeres han llegado a colocar de manifiesto su interés, por ejemplo, en el aborto legal, por ser de libre decisión cuantos hijos se desea tener (Zárate Cuello, 2014). Estos avances se han generado luego de diversas disputas entre sectores antagónicos como lo son los movimientos latinoamericanos que buscan la aceptación de los DSR y el catolicismo o movimientos conservadores (Morán Faúndes \& Peñas Defago, 2013).

Investigaciones realizadas por Baldivieso (2010) en Bolivia muestran el panorama que es común en las sociedades, especialmente en las latinoamericanas. Mujeres expuestas a familias $y$ sociedades patriarcales que vulneran con frecuencias los derechos de la mujer, incluidos los DSR. Junto a esto presentan deterioro físico y emocional por ser abusadas sexualmente, por no ejercer su libertad, por no decidir autónomamente el querer o no reproducirse, y como consecuencia de esto último la existencia de embarazos no deseados, abortos espontáneos o practicados que atentan contra la integridad de la persona como ser humano. Es por tal razón que deciden darles voz a estas mujeres, abriéndoles las puertas para que salgan de ese encierro, de esa oscuridad, la cual han vivido por tanto tiempo.

En distintos países latinoamericanos como Colombia, Cuba, Argentina, y como ya se ha comentado, históricamente no había un fácil acceso a la educación y a la información, y en la familia tradicional católica, temas como la formación de la vida en torno a la sexualidad y la reproducción no podían ser discutidos, se le asignaba esta responsabilidad a las mujeres, además que se tenía la concepción de que las familias extensas eran consideradas de un alto status a nivel social y sustento económico favorable (Alfonso Fraga, 2006). Sin embargo, en muchos lugares los DSR han sido motivo de luchas por las mujeres tanto en el ámbito público, como en el ámbito privado, más puntualmente en la reclamación de cuántos, cuándo y cómo tener hijos. En ese sentido se pone de manifiesto el vertiginoso desarrollo de los modelos de familia y las libertades que adquieren las mujeres gracias al reconocimiento que tienen los DSR (Caneva, 2015).

Viendo los derechos reproductivos como los derechos con los que cuenta la mujer para poder regular su sexualidad y capacidad productora, y exhiben al hombre como un ser responsable de las consecuencias del ejercicio de su sexualidad; es necesario que el poder sobre el cuerpo sea autónomo, de forma que la mujer sea consciente de no permitir imposiciones sobre su cuerpo (Bonaccorsi \& Reybet, 2008).

En las últimas décadas, las mujeres con un arduo esfuerzo por trascender ese papel de subordinación a través de procesos sociales y políticos y de ocupar espacios en las esferas relacionadas con la masculinidad cotidianamente, se ha empezado a mejorar su posición en el entorno social y cultural. Se está viendo a la mujer como un nuevo sujeto social, que ha superado la etapa de las denuncias y ha logrado llegar al diseño de las estrategias y propuestas que inciden en las agendas públicas 
y amplían la ciudadanía de las mujeres, lo que produjo reformas que continúan teniendo una deuda con la práctica y ejercicio continuo en la vida cotidiana (Sagot Rodríguez, 2010).

En distintos países los DSR han tenido gran espacio dentro de la discusión pública para alcanzar escenarios de reconocimiento. Por ejemplo, en los Estados Unidos el aborto hace parte de los DSR que tienen las mujeres; en Colombia, en cambio, este es aceptado solo en tres situaciones exclusivas: en acceso carnal violento, malformaciones y cuando está en riesgo la salud de la madre (Zárate Cuello, 2014). Aquí el debate continúa abierto entre los DSR de las mujeres y los derechos del niño por nacer, sin perder de vista la carga moral que tiñe la discusión (Mayorga Madrigal, 2016; Valdés Díaz, 2012; Zicavo et al., 2015).

No obstante, sobre el papel de la mujer y los DSR, la Conferencia El Cairo realzó su empoderamiento y la mejora de la situación de las niñas, conjuntamente con el derecho a la salud sexual y reproductiva, como parte de la estrategia para la reducción de la pobreza, mejora de la salud y la calidad de vida. Sin embargo, sobre el aborto enfatiza en no promoverlo como método de planificación familiar (Galdos Silva, 2013).

Las críticas al discurso tradicional de los derechos humanos provenientes del feminismo evidencian la prevalencia de un discurso androcéntrico que reconoce como modelo de titularidad de derechos al varón adulto. Sólo recientemente se introduce la perspectiva de género y se evidencia lo que ocurre en el ámbito privado, es decir los aspectos sexuales y reproductivos (Badilla, 2005, 2006; Romero Ricalde, 2007; Taus, 2014). Ellas han resistido, sus subjetividades les han contribuido a la autoafirmación, empoderamiento y autonomía para resistir y legitimar otras construcciones sociales sobre ser mujer (Zaldúa, Pawlowicz, Longo, \& Moschella, 2010). De ahí la necesidad de incorporar el enfoque de género de manera progresiva para reconstruir el saber sobre la salud sexual y reproductiva desde la mirada particularizante de hombres y mujeres, lo que conllevaría a la reformulación de políticas públicas y el reconocimiento de la diversidad sexual tan necesarias para el pleno goce $y$ ejercicio de los DSR (Cruz Pérez, 2015; Ospina Muñoz \& Castaño López, 2009).

\section{Los Jóvenes y Adolescente y los DSR}

El ser humano en su proceso de desarrollo vivencia diferentes etapas, la adolescencia es una de ellas, la cual está llena de cambios biopsicosociales en los que se encuentra involucrada la sexualidad y la reproducción que, en caso de no ser orientada de manera adecuada, los adolescentes podrían estar expuestos a factores de riesgo en su ambiente social y más aún si pertenecen a sectores de pobreza y escasa educación.

La preocupación sobre los derechos de la infancia y la adolescencia por parte de los organismos internacionales no se hace esperar. Es de anotar que, pese a dichas proclamas normativizadas a escala internacional, en los gobiernos suramericanos no hay un total cumplimiento. Según Flórez \& Soto (2008):

el conocimiento de temas relacionados con la SSR [salud sexual y salud reproductiva] indica vacíos importantes en todos los países en cuanto a la fisiología de la reproducción - y además un desconocimiento en los países de transición rezagada -en cuanto a los métodos y su fuente de suministro, especialmente entre las mujeres menos favorecidas socialmente: las menos educadas y las rurales. (p. 18)

La convención (CIDN) postula que pese a los derechos de autonomía que debe gozar este grupo etario, se permite un acompañamiento por parte de los padres sinónimo de dirección y orientación apropiadas al niño, teniendo en cuenta la evolución de sus facultades, edad y madurez. Es importante señalar que según la OMS la adolescencia como el periodo en que el ser humano está apto para reproducirse y además está en un transitar psicológico del periodo de niñez a la adultez, en consecuencia, comienza a adquirir su responsabilidad económica con miras a una independencia (Valenzuela Rivera \& Casas Becerra, 2007). Es 
en este momento de la vida del ser humano en donde se enfrenta a grandes cambios, entre ellos la lucha por su gestión sexual. Por ello es un momento importante para profundizar y darle a conocer sus DSR.

Frecuentemente adolescentes y jóvenes carecen de conocimientos sobre sus propios DSR y tampoco a dónde acudir en caso de que estos les sean vulnerados, así mismo desconocen sobre conductas sexuales seguras (Roncal-Cabrera \& Carranza-Salas, 2012). Esta ausencia se suma a la necesidad de formarse como personas que sienten, se emocionan, valoran y no solo que reciben conocimiento elaborado y ajustado a las necesidades de los adultos. Las estrategias de potenciación del amor son el mejor camino de transformación de la sociedad, ya que cada joven desea ser él mismo, olvidándose del exterior y reencontrándose con su identidad (Valencia Jiménez \& Solera Martínez, 2009).

Sin embargo, se evidencian falencias a nivel familiar y escolar, al transmitir la sexualidad desde referentes biologicistas y no tener en cuenta lo que los jóvenes desean aprender como, por ejemplo, interpretar sus emociones y sentimientos, a cultivar el amor en todas sus formas y manifestaciones, el saber qué sienten o experimentan los seres humanos en los diferentes ciclos de la vida. Pero para lograr esto, se requiere que los adultos aprendan a dejar sus prejuicios infundados por temores, creencias, mitos o tabúes y que se han interiorizado por el rol cultural que se perpetuando en patrones y normas (Molina-Berrío \& Posada-Zapata, 2013; Valencia Jiménez \& Solera Martínez, 2009).

También parte de la visión dominante del adulto que problematiza al adolescente y al joven es la visión generalizada de concebirlos como personas sin responsabilidad, propensa a eventos contrarios a la norma (Molina-Berrío \& PosadaZapata, 2013). Dejando a un lado la posibilidad de empoderarlos y ofrecerles el acompañamiento para el reconocimiento de sus DSR y con ello a la prevención de situaciones que incluso puedan afectar su vida como, por ejemplo, las infecciones por trasmisión sexual, que en el caso más grave el VIH (Valdés Díaz, 2012).
Es de vital importancia fortalecer la autonomía, para que la población adolescente actúe desde el conocimiento de sí misma, conciencia sobre su propio cuerpo y tome decisiones que favorezcan un estilo de vida saludable y una mejora en la calidad de vida (Arias Arroyo et al., 2011).

\section{Los DSR en los Escenarios Educativos}

La necesidad de diseñar e implementar diversos programas y estrategias de educación sobre DSR dirigidos con mayor énfasis a la población estudiantil (niños y adolescentes) y docentes con el fin de favorecer un entorno educativo que permita brindar información que ayude a realizar una buena toma de decisiones en torno a la sexualidad sigue aún vigente (Preinfalk-Fernández, 2015).

Estudios realizados por Niño Bautista et al. (2012) en Colombia, con adolescentes de colegio entre 12-19 años de edad demuestran que la psicoeducacion sobre los DSR disminuye en gran medida los riesgos en las practicas sexuales, como embarazos no deseados, y ETS e incrementa el conocimiento y formacion para la proteccion y el respeto de los derechos sexuales y reproductivos. Pero para obtener estos resultados, tambien se requiere que los profesores se capaciten en estos temas y apropien estratégias pedagógicas que favorezcan la calidad de la educación en DSR (Mantilla Uribe, Hakspiel Plata, Rincón Méndez, Smith Hernández, \& Hernández Quirama, 2012; Ospina Muñoz \& Castaño López, 2009). Para ello, una herramienta que acerca a profesores al entorno de sus estudiantes es la web que para el caso de los DSR se encuentran muchos que permiten informar y construir sentidos alternos para su apropiación (Chen, 2012).

En la construcción de la educación sexual, hay aspectos macro que son evidentes en su influencia sobre las personas y la manera de ejercer los DSR, pero hay otros que podrían pasar desapercibidos e incluso ser tomados como algo normal. Desde la niñez, una gran parte de la población asiste a centros educativos, todos ocupan un lugar en su familia, entre sus amigos, etc., pero es allí, en esa socialización donde se tiende a reproducir modelos que a 
veces resultan ser destructivos, de sumisión y jerárquicos, lo cual afecta directamente en su ejercicio de sus DSR (Climent, 2009).

Una de las consecuencias más evidentes de la falta de una adecuada educación sexual y reproductiva es la gran cantidad de embarazos que se producen en adolescentes, situación aún más precoz cuando prevalecen condiciones de pobreza y baja calidad de educación o falta de ella (del Castillo Matamoros, Roth Deubel, Wartski Patiño, Rojas Higuera, \& Chacón Barliza, 2008).

Por último, es importante que en los países en desarrollo se generen campañas pedagógicas en donde las costumbres tradicionales sean transformadas para alcanzar el bienestar de las personas con respecto a la sexualidad y la reproducción, con el fin de acabar el tabú sobre esta última y así poder visibilizar los DSR como garantes principales de la salud sexual y reproductiva (García Suárez, 2007). Las políticas de salud pública y educación deben logran impactar de una forma significativa en la salud sexual y reproductiva (del Castillo matamoros et al., 2008).

\section{Conclusiones}

A partir de la revisión documental se establece que el reconocimiento de los derechos sexuales y reproductivos sienta sus bases en la Conferencia Mundial de los Derechos Humanos celebrada en Teherán (1968), fue surgiendo en la medida en que se promovían y reconocían los derechos humanos y las distintas conferencias internacionales para promover los derechos de la mujer. Fue en la Conferencia Internacional de población y desarrollo celebrada en el Cairo en 1994 donde se reconocen los DSR y desde entonces se generan una serie de encuentros y conferencias en Suramérica que lleva a incorporar en la constitución de estos países los DSR.

La sexualidad, los derechos sexuales y derechos reproductivos han estado influenciados y afectan la sociedad, la economía, la política y la cultura, en este sentido el ejercicio de los DSR no ha sido nada fácil, ha implicado desafiar el horizonte cultural en América latina. Las políticas regionales patriarcales y la religión católica han sido un verdadero obstáculo para reconocer en las personas los DSR. A pesar de estas tendencias, los movimientos feministas han venido gestando fuertes luchas y movimientos sociales que han llevado a que se le reconozca los DSR en los países Latinoamericanos y se implementen políticas públicas orientadas a promover la inclusión, donde se perciba a la mujer como un sujeto social de derechos.

Es en la adolescencia, cuando el ser humano tiene que enfrentarse a grandes cambios dentro de los cuales esta su gestión por la sexualidad, donde debe recibir una educación que le permita formarse como persona que siente, se emociona, valore y que sea ajustada a sus necesidades donde fortalezca su autonomía, que le permita tomar conciencia sobre su propio cuerpo y tome decisiones que favorezcan estilos de vida saludables y en consecuencia disminuir los riesgos propios de las prácticas sexuales como el embarazo precoz y ETS.

\section{Referencias Bibliográficas}

Alfonso Fraga, J. C. (2006). El descenso de la fecundidad en Cuba: De la primera a la segunda transición demográfica. Revista Cubana de Salud Publica, 32(1), 1-19. Recuperado a partir de http://scielo.sld.cu/ pdf/rcsp/v32n1/spu02106.pdf

Arias Arroyo, A., Campos Masís, R., Chaves Arce, M., García Segura, M., Mora Lobo, D., Ramírez Campos, J., ... Vega Mejías, J. (2011). Maternidad adolescente: Herramientas para una mejor calidad de vida. Medicina Legal de Costa Rica, 28(1), 25-30. Recuperado a partir de http://www. scielo.sa.cr/pdf/mlcr/v28n1/art3v28n1.pdf

Badilla, A. E. (2005). Marco jurídico para la prevención del VIH/SIDA con jóvenes en Costa Rica: análisis de la legislación costarricense sobre juventud, VIH/SIDA y migración. Recuperado a partir de http://www. portalsida.org/repos/costa rica.pdf 
Badilla, A. E. (2006). VIH, SIDA y derechos humanos: retos de política y legislación. En Reunión de expertos sobre población, desigualdades y derechos humanos. Santiado de Chile: CEPAL - Naciones Unidas. Recuperado a partir de http://www. cepal.org/sites/default/files/events/files/ badillaae.pdf

Baldivieso, D. (2010). Abriendo el paquete envuelto. Investigación acción sobre derechos sexuales y reproductivos en El Alto. T'inkazos, (28), 99-107. Recuperado a partir de http://www.scielo.org.bo/pdf/ rbcst/v13n28/v13n28a06.pdf

Bareiro, L. (2003). Los derechos reproductivos $\mathrm{y}$ los derechos humanos universales. En Promoción y defensa de los derechos reproductivos: nuevo reto para las instituciones nacionales de derechos humanos (pp. 119-133). San José, C.R: Instituto Interamericano de Derechos Humanos. Recuperado a partir de https://www.iidh.ed.cr/IIDH/ media/1832/promocion-y-defensa-dederechos-reproductivos-espanol-2006.pdf

Bedoya Abella, C. L. (2014). Educación para la sexualidad y construcción de ciudadanía. Sophia, 10(1), 95-106. Recuperado a partir de http://www.scielo.org.co/pdf/sph/ v10n1/v10n1a09.pdf

Bonaccorsi, N., \& Reybet, C. (2008). Derechos sexuales y reproductivos: un debate público instalado por mujeres. LiminaR. Estudios Sociales y Humanísticos, 6(2), 5264. Recuperado a partir de http://www. redalyc.org/articulo.oa?id=74511194004

Campero, L., Kendall, T., Caballero, M., Mena, A. L., \& Herrera, C. (2010). El ejercicio de los derechos sexuales y reproductivos: Un estudio cualitativo de personas heterosexuales con VIH en México. Salud Publica de Mexico, 52(1), 61-69. http://doi. org/10.1590/S0036-36342010000100010

Caneva, H. A. (2015). Planificación familiar y modelo conyugal en Argentina (1960-
1970). Reflexiones sobre la matriz de los derechos sexuales y reproductivos. Trabajos y Comunicaciones, (42). Recuperado a partir de http://www.trabajosycomunicaciones. fahce.unlp.edu.ar/article/view/ TyC2015n42a08/6927

Carbonelli, M. (2015). Valores para mi País: Evangélicos en la Esfera Política Argentina (2008-2011). Dados, 58(4), 981-1015. http:// doi.org/10.1590/00115258201564

Carreño Ruiz, P. J., Artunduaga Alpire, Y., Suarez Barrientos, E. L., \& Cornejo Bascopé, G. (2007). Interpretación bioética de la ley de derechos sexuales y reproductivos. Revista Científica Ciencia Médica, 10(1), 4849. Recuperado a partir de http://www. redalyc.org/pdf/4260/426041216017.pdf

Chen, Y.-Z. (2012). Análisis de los sitios web del movimiento por los derechos sexuales y reproductivos en México. Sociedade $e$ Cultura, 15(1), 87-98. http://doi.org/10.5216/ sec.v15i1.20675

Ciriza, A. (2002). Consenso y desacuerdo. Los derechos reproductivos y sexuales como derechos ciudadanos de las mujeres en Argentina. El Catoblepas. Revista Crítica del Presente, (9), 18. Recuperado a partir de http://www.nodulo.org/ec/2002/n009p18. htm

Climent, G. I. (2009). Entre la represión y los derechos sexuales y reproductivos: socialización de género y enfoques de educación sexual de adolescentes que se embarazaron. La Ventana, (29), 236-275. Recuperado a partir de http://www.scielo. org.mx/pdf/laven/v3n29/v3n29a10.pdf

Corrêa, S., \& Petchesky, R. (1996). Direitos Reprodutivos uma Perspectiva Feminista. Phyisis: Revista de Saúde Coletiva, 6(1/2), 147-177. http://doi.org/10.1590/S010373311996000100008

Cosse, I. (2008). Familia, sexualidad y género en los años 60. Pensar los cambios desde 
la Argentina: desafíos y problemas de investigación. Temas Y Debates, 16(Diciembre), 131-149. Recuperado a partir de http://rephip.unr.edu.ar/ handle/2133/1547

Cruz Pérez, M. del P. (2015). Acceso a derechos sexuales y reproductivos de las mujeres con discapacidad: el papel de las y los prestadores de servicios. Revista Estudios de Género. La ventana, (42), 7-45. Recuperado a partir de http://www. revistascientificas.udg.mx/index.php/LV/ article/view/5712/5234

del Castillo matamoros, S., Roth Deubel, A. N., Wartski Patiño, C. I., Rojas Higuera, R., \& Chacón Barliza, O. A. (2008). La implementación de la política pública de salud sexual y reproductiva (SSP) en el Eje Cafetero colombiano: el caso del embarazo adolescente. Revista Latinoamericana de Ciencias Sociales, Niñez y Juventud, 6(1), 217255. Recuperado a partir de http://www. redalyc.org/articulo.oa?id $=77360108$

Flórez, C. E., \& Soto, V. E. (2008). El estado de la salud sexual y reproductiva en América Latina y el Caribe: Una visión global. Banco Interamericano de Desarrollo (BID). Washington D.C.: Banco Interamericano de Desarrollo. Recuperado a partir de http:// www.iadb.org/res/publications/pubfiles/ pubWP-632_esp.pdf

Galdos Silva, S. (2013). La conferencia de El Cairo y la afirmación de los derechos sexuales y reproductivos, como base para la salud sexual y reproductiva. Revista Peruana de Medicina Experimental y Salud Publica, 30(3), 455-460. Recuperado a partir de http:// www.scielosp.org/pdf/rpmesp/v30n3/ a14v30n3.pdf

García Suárez, C. I. (2007). Diversidad sexual en la escuela. Dinámicas pedagógicas para enfrentar la homofobia. Colombia Aprende (1.a ed.). Bogotá: Colombia Diversa. http://doi. org/10.1590/S0102-46982012000300007
González,A.C.(2016).Autonomía física, derechos sexuales y reproductivos. En Decimotercera Conferencia Regional sobre la Mujer de América Latina y el Caribe. Montevideo: Naciones Unidas, CEPAL. Recuperado a partir de http://conferenciamujer.cepal.org/sites/ default/files/presentations/panel_3_-_ana_ cristina_gonzalez.pdf

González, A., \& Victoria, M. I. (2012). De lo rural a lo urbano: tensiones entre tradición y modernidad en una comunidad afrodescendiente del municipio de Carrito, Valle del Cauca, Colombia. Revista nodo, 6(12), 55-66. Recuperado a partir de https://dialnet.unirioja.es/descarga/ articulo/4014060.pdf

González, D. (2009). Las construcciones sociales de la salud sexual entre los jóvenes inmigrantes mexicanos en los Estados Unidos. Revista Iberoamericana de Juventud, 9, 1-52. Recuperado a partir de http://www. oij.org/file_upload/publicationsItems/ document/Revista1264153378.pdf

Hernández-Rosete, D., García, O. M., Bernal, E., Castañeda, X., \& Lemp, G. (2008). Migración y ruralización del SIDA: relatos de vulnerabilidad en comunidades indígenas de México. Revista de saude publica, 42(1), 131-138. $\quad$ http://doi.org/10.1590/S003489102008000100017

Instituto Colombiano de Bienestar Familiar, \& Profamilia. (2008). Módulo Conceptual. Derechos Sexuales y Reproductivos. Bogotá: Imprenta Nacional de Colombia.

Jones, D. E., \& Carbonelli, M. A. (2012). Evangélicos y derechos sexuales $\mathrm{y}$ reproductivos: actores y lógicas políticas en la Argentina contemporánea. Ciências Sociais Unisinos, 48(3), 225-234. http://doi. org/10.4013/csu.2012.48.3.05

Mantilla Uribe, B. P., Hakspiel Plata, M. C., Rincón Méndez, A. Y., Smith Hernández, D., \& Hernández Quirama, A. (2012). Promoción de derechos sexuales y 
reproductivos en adolescentes de Bucaramanga, Floridablanca y Lebrija Colombia. Revista Salud UIS, 44(3), 13-23. Recuperado a partir de http://www.scielo. org.co/pdf/suis/v44n3/v44n3a03.pdf

Mayorga Madrigal, C. (2016). Construcción ética de los derechos sexuales y reproductivos. Sincronía. Revista de Filosofía y Letras, (69), 3237. Recuperado a partir de http://sincronia. cucsh.udg.mx/pdf/69/mayorga_69.pdf

Miller, A. (2002). Las demandas por derechos sexuales. En III Seminario Regional Derechos Sexuales, Derechos Reproductivos, Derechos Humanos (pp. 121-140). Lima: Cladem.

Molina-Berrío, D. P., \& Posada-Zapata, I. C. (2013). Perspectiva de algunos actores sobre los modelos de educación para la salud subyacentes en programas de salud sexual y reproductiva dirigidos a adolescentes, Medellín 2004-2007. Revista Facultad Nacional de Salud Pública, 31(1), 19-29. Recuperado a partir de http://www. scielo.org.co/pdf/rfnsp/v31n1/v31n1a03. pdf

Montaño, S., \& Aranda, V. (2006). Reformas constitucionales y equidad de género. Informe final, Seminario internacional. Santiago de Chile: CEPAL - Naciones Unidas. Recuperado a partir de http:// repositorio.cepal.org/bitstream/ h and le/11362/6827/S0500089. es.pdf? sequence $=1 \&$ isAllowed $=y$

Morán Faúndes, J. M., \& Peñas Defago, M. A. (2013). ¿Defensores de la vida? ¿De cuál «vida»? Un análisis genealógico de la noción de «vida» sostenida por la jerarquía católica contra el aborto. Sexualidad, Salud y Sociedad, (15), 10-36. http://doi.org/10.1590/ S1984-64872013000300002

Niño Bautista, L., Hakspiel Plata, M. C., Rincón Méndez, A. Y., Aragón Borré, D., Roa Diaz, Z. M., Galvis Padilla, D. C., \& Luna, A. (2012). Cambios persistentes en conicimientos, actitudes y prácticas sobre sexualidad en adolescentes y jóvenes escolarizados de cuatro municipios de Santander - Colombia. Revista Salud UIS, 44(2), 21-33. Recuperado a partir de http://www.scielo.org.co/pdf/suis/v44n2/ v44n2a05.pdf

Ospina Muñoz, D. E., \& Castaño López, R. A. (2009). Producción científica sobre derechos sexuales y reproductivos, Colombia 1994-2004. Investigación y Educación en Enfermería, 27(2), 181-190. Recuperado a partir de http://www.redalyc.org/articulo. oa?id=105213195003

Pacheco-Sánchez, C. I., Rincón-Suárez, L. J., Guevara, E. E., Latorre-Santos, C., Enríquez-Guerrero, C., \& Nieto-Olivar, J. M. (2007). Significaciones de la sexualidad y salud reproductiva en adolescentes de Bogotá. Salud Publica de Mexico, 49(1), 4551. Recuperado a partir de http://www. scielosp.org/pdf/spm/v49n1/a07v49n1.pdf

Petracci, M., \& Pecheny, M. (2009). Panorama de derechos sexuales y reproductivos, Argentina 2009. Argumentos. Revista de crítica social, (11). Recuperado a partir de http://biblioteca.clacso.edu.ar/Argentina/ iigg-uba/20100331025726/3.pdf

Preinfalk-Fernández, M. L. (2015). Desafíos de la formación docente en materia de educación sexual. Revista Electrónica Educare, 19(1), 85-101. http://doi.org/http:// dx.doi.org/10.15359/ree.19-1.5

Reartes, D. L. (2011). La comunidad y la ciudad como referentes en la construcción social de riesgos frente al VIH-Sida entre jóvenes estudiantes hablantes de lenguas indigenas de los Altos de Chiapas. Desacatos, (35), 5974. Recuperado a partir de http://www. scielo.org.mx/pdf/desacatos/n35/n35a5.pdf

Romero Ricalde, L. (2007). Jóvenes: sus derechos sexuales y reproductivos. Chacarera, (34), 18-21. Recuperado a partir de http:// biblioteca.clacso.edu.ar/Peru/cmp-floratristan/20120823010740/impacto34.pdf 
Roncal-Cabrera, D. J., \& Carranza-Salas, M. Y. (2012). Conocimiento y percepción sobre derechos sexuales y reproductivos de los estudiantes de la Universidad Privada Antenor Orrego. Revista del Cuerpo Médico Hospital Nacional Almanzor Aguinaga Asenjo, 5(3), 7-12. Recuperado a partir de https://dialnet.unirioja.es/servlet/ articulo?codigo $=4040351$

Sagot Rodríguez, M. (2010). Demandas desde la exclusión: representatividad democrática y cuotas de participación política en Costa Rica. Revista de Ciencias Sociales, 4(130), 2943. Recuperado a partir de http://www. redalyc.org/pdf/153/15321318002.pdf

Staff Wilson, M. (1998). Mujer y Derechos Humanos. Koaga Ronéeta. Panamá. Recuperado a partir de http://www. derechos.org/koaga/viii/staff.html

Straw, C., \& Mattioli, M. (2013). Entre el discurso legal y las opiniones de las mujeres: Reflexiones sobre la apropiación subjetiva de derechos sexuales y reproductivos. Revista Pilquen, 16(2), 1-11. Recuperado a partir de http://www.scielo.org.ar/pdf/ spilquen/v16n2/v16n2a02.pdf

Taus, P. A. (2014). La igualdad de género y el acceso a la justicia de las mujeres víctimas de violencia en la región dentro del sistema interamericano de protección de los derechos humanos. Revista IUS, 8(34), 21-41. Recuperado a partir de http://www. scielo.org.mx/pdf/rius/v8n34/v8n34a3.pdf

Távara Orozco, L. (2016). Contribución de la Revista Peruana de Ginecología y Obstetricia al desarrollo de los derechos sexuales y reproductivos en Perú. Revista Peruana de Ginecología y Obstetricia, 62(1), 31-57. Recuperado a partir de http://www. scielo.org.pe/pdf/rgo/v62n1/a04v62n1.pdf

Valdés Díaz, C. del C. (2012). Del derecho a la vida y los derechos sexuales y reproductivos, ¿configuración armónica o lucha de contrarios? IUS. Revista del Instituto de
Ciencias Jurídicas de Puebla A.C., 6(29), 216239. Recuperado a partir de http://www. redalyc.org/pdf/2932/293222991012.pdf

Valencia Jiménez, N. N., \& Solera Martínez, N. F. (2009). Necesidades de aprendizaje para la sexualidad de jóvenes entre 10 y 19 años en el caribe colomnbiano. Investigación y Desarrollo, 17(1), 106-131. Recuperado a partir de http://www.scielo.org.co/pdf/ indes/v17n1/v17n1a05.pdf

Valenzuela Rivera, E., \& Casas Becerra, L. (2007). Derechos sexuales y reproductivos: Confidencialidad y VIH/SIDA en adolescentes chilenos. Acta Bioethica, 13(2), 207-215. http://doi.org/10.4067/S1726569X2007000200008

Villanueva Flores, R. (2006). Protección constitucional de los derechos sexuales y reproductivos. Revista IIDH, 43, 391-450. Recuperado a partir de http://www.scielo. org.ar/pdf/spilquen/v16n2/v16n2a02.pdf

Zaldúa, G., Pawlowicz, M. P., Longo, R., \& Moschella, R. (2010). Derechos sexuales y reproductivos de las mujeres de la CABA. Obstáculos y resistencias. Anuario de investigaciones, 17, 267-275. Recuperado a partir de http://www.scielo.org.ar/pdf/ anuinv/v17/v17a26.pdf

Zárate Cuello, A. de J. (2014). ¿Es el aborto un derecho sexual y reproductivo de la mujer? Análisis desde el bioderecho, la Bioética, la biopolítica y la biojurídica en Estados Unidos, España y Colombia. Revista Latinoamericana de Bioética, 14(2), 12-27. Recuperado a partir de http://www. redalyc.org/articulo.oa?id=127032031002

Zicavo, E., Astorino, J., \& Saporosi, L. (2015). Derechos sexuales y reproductivos en Argentina: Los proyectos parlamentarios referidos al aborto. Revista Reflexiones, 94(2), 89-99. Recuperado a partir de http:// revistas.ucr.ac.cr/index.php/reflexiones/ article/view/25459/25711 